\title{
Spatial Demogenetic Model for Studying Phenomena Observed upon Introduction of the Ragweed Leaf Beetle in the South of Russia
}

\author{
Yu.V. Tyutyunov ${ }^{1,3} *$, O.V. Kovalev ${ }^{2}$, L.I. Titova ${ }^{3}$ \\ ${ }^{1}$ Institute of Arid Zones, Southern Scientific Centre of the Russian Academy of Sciences \\ Chekhov street, 41, 344006 Rostov-on-Don, Russia \\ 2 Zoological Institute of the Russian Academy of Sciences \\ University Quay, 1, 199034 Saint Petersburg, Russia \\ ${ }^{3}$ Vorovich Research Institute of Mechanics and Applied Mathematics, Southern Federal University \\ Stachki street, 200/1, 344090 Rostov-on-Don, Russia
}

\begin{abstract}
The introduction of the ragweed leaf beetle in the South of Russia in 1978-1989 was accompanied by a number of spectacular phenomena that determined the general success of the ragweed control and further dispersal and acclimatization of the beetles: $(i)$ formation of solitary population waves (SPW), characterized by an extremely high density of the phytophage population at the narrow band of the front of a moving wave defoliating nearly all ragweed plants, and (ii) rapid, within 5-6 generations, development of flight in the leaf beetle species that in its homeland lost the ability to fly. We present here a demogenetic model capable of reproducing both these phenomena, assuming that the flight ability of a phytophage population is governed by a single diallelic locus with flight and flightless alleles that determine three genotypes of the ragweed leaf beetle. Simulation results agree well with the practical recommendation of retaining a high density of common ragweed in the release area in order to provide the necessary conditions for the initial increase of the leaf beetle population and the formation of the wave. The model confirms the earlier hypothesis that the SPW is the key factor that determines efficiency of weed biocontrol program. We demonstrate also that the formation of the wave has crucially accelerated the development of the beetles' ability to fly.
\end{abstract}

Keywords and phrases: spatio-temporal dynamics, trophic system, Allee effect, taxisdiffusion-reaction, trophotaxis, demogenetic Kostitzin model, gene surfing, biological control, weed control, Ambrosia artemisiifolia L., Zygogramma suturalis $\mathrm{F}$.

Mathematics Subject Classification: 92B05, 92D25, 92D50, 92D40

\section{Introduction}

The ragweed leaf beetle, Zygogramma suturalis F. was imported from North America and introduced to the Old World in 1978 by O.V. Kovalev [33], as a biological control agent against the common ragweed Ambrosia artemisiifolia L. The complete program of introduction and acclimatization of $Z$. suturalis, led

\footnotetext{
${ }^{*}$ Corresponding author. E-mail: tyutyunov@sfedu.ru
} 
by the Zoological institute of the Academy of sciences of the USSR was carried out in the territories of 16 regions of the former Soviet Union from Ukraine to the Far East, while the most intensive works took place in the South of Russia (the Stavropol Territory, the Rostov Region, the Krasnodar Territory, etc.) during the 1978-1989 period. The introduction halted the explosion phase of common ragweed invasion in the South of Russia that was induced by the large scale disturbances of soils and landscapes on account of hard-fought battles for these territories during the Second World War [33,35].

Field observations of the spreading of the leaf beetle allowed discovering a new, previously unknown phenomenon of the formation of population autowaves with extremely high density of Zygogramma suturalis (up to 5000 individuals per $m^{2}$ at the wave front). The advance of the wave causes virtually the complete destruction of common ragweed plants. Such behaviour is atypical for this species - the ragweed leaf beetle never creates dense aggregations within its native habitats. Kovalev and Vechernin $[33,36,37]$ proposed an original mathematical model describing the movement of such a wave of phytophagous insect species, and coined the term "solitary population wave" (SPW) which stresses the soliton-like shape of the wave profile and the necessity of reaching some critical level of population density to provoke an SPW formation (hereafter, we use the term SPW as defined in [36,37]). Interestingly, in very different cases of successful programs of phytophagous insect species introductions to combat weeds, the spatio-temporal dynamics of trophic systems follow qualitatively the same pattern typical of SPW propagation: suppression of St. Jones wort (Hypericum perforatum L.) by the leaf beetle Chrysolina quadrigemina (Suffrian) on the American continents [21,24], successful control of the floating weed Salvinia molesta Mitchell by the salvinia weevil (Cyrtobagous salviniae Calder and Sands) released into the water basins in Australia, Africa, India and Oceania $[24,52,53]$, etc. However entomologists did not attach any importance to the role played by the wave regimes in these and similar cases. The first time that the investigation of the formation of an SPW of a phytophage agent as a key factor determining the efficiency of weed biocontrol occurred in the frameworks of the program of acclimatization of the ragweed leaf beetle in the North Caucasus $[34,36,37]$. Later this concept as well as the Kovalev-Vechernin model [36,37] of SPW were effectively applied for biological control of exotic saltcedars (Tamarix spp.) by tamarisk beetles (Diorhabda spp.) in North America [42].

In various successful campaigns for weed biocontrol on different continents, the initial phase of the formation of a phytophagous population wave can clearly be distinguished from the rest of the process: an enormously high population abundance of up to tens of millions of individuals can be reached within 4-6 generations only [34,53]. Obviously this accumulation phase requires the availability of more or less homogeneous area-extensive plots with a high biomass of host plant population. Accordingly, the recommended method for Zygogramma suturalis application consists in a preliminary design of plots (about 2 ha) with high density of the ragweed (e.g., by plowing the soil), that will become the sources of the phytophage population waves' formation [37]. It is emphasised [34-37] that studies on SPW has an evident practical importance. Generally speaking, without considering wave dynamics of a weedphytophage system, any reasoning on a control agent efficiency makes little sense. This principle agrees with a number of theoretical results emphasising the crucial role of spatio-temporal heterogeneity and consumer's spatial behaviour in achieving sustainable biological control and stabilisation of trophic system $[11,17,40,43,48,55,65]$.

Ecological and demographic processes as well as evolution transformations in population undergoing spatially heterogeneous wave regime differ from what might be expected for homogeneously well-mixed case of population dynamics. In particular, with the help of a spatial demogenetic model, Tyutyunov and coauthors $[67,68]$ demonstrated that spatial heterogeneity can cause violations of the Hardy-Weinberg conditions, considerably delaying the evolution of resistance to the Bacillus thuringiensis toxins in an insect pest population. Another interesting effect related to the population wave propagation is the socalled "gene surfing" phenomenon that can facilitate the dispersal of mutations arising in the wave front of an expanding invader population $[9,18,39]$. Regarding the events observed during the introduction to the Old World of the ragweed leaf beetle, we believe that these wave effects provoked the reported unusual variation of the phenotype of Zygogramma suturalis in SPW, manifesting itself in the uncommon 
variety of the color pattern, including melanistic forms of imago, which do not even occur in other species of the genus $[32,34,41]$. Another phenomenon related to the fixation of beneficial mutations in the wave front of $Z$. suturalis was the spectacularly rapid development of flight in the leaf beetle species that has in its homeland lost the ability to fly $[32,34,36,37]$. Within just 5-6 generations, flightless beetles were completely replaced by the 'flyers'. According to observations [32], formation of 'flyers' had only taken place under the strong selection resulting from the high population density within the front of the expanding wave of the ragweed leaf beetle population.

Though the Kovalev-Vechernin model [33,36,37] describes well the initial formation, and further movement of a single solitary population wave of phytophage population, it is not intended for long-term simulation of the dynamics of the trophic system at large spatio-temporal scale. Besides, being designed for different purposes, this model does not take into account evolutionary changes of an invading species.

In the present paper we propose a simulation model of a spatially distributed system "plant - consumer", taking into account both the genetic structure, and the spatial behaviour of the ragweed leaf beetles. We show that with reasonable parameter values, the model can naturally reproduce the observed pattern of common ragweed suppression, as well as the rapid development of flight in the leaf beetle population.

\section{The demogenetic model}

We construct spatial demogenetic models based on a taxis-diffusion-reaction system of partial differential equations (PDEs) $[7,8,45,46,48,50]$, with the Kostitzin-type reproduction terms [27-30] used to describe the local kinetics of competitive genotypes of the consumer (i.e., of the ragweed leaf beetle). The random movements of insects within the habitat is described by diffusion terms while tactic (advective) terms formalise the directed movements of beetles, stimulated by heterogeneities in the plant biomass distribution (trophotaxis). Thus, in order to describe the spatial movements of phytophage we use the earlier proposed approach to modelling animal taxis $[16,55,63,64]$, that naturally generalises the basic principles of the classical Keller-Segel chemotaxis model [25] to the case of two-component trophic system (see $[64,66])$.

Let's describe the model in more details.

\subsection{Model assumptions and requirements}

The list of specific requirements for the model includes the following items:

- explicit description of spatial dynamics;

- considering both random (diffusion) and active directed movements of phytophage population density, stimulated by heterogeneities in the food resource (trophotaxis);

- accounting for spatial heterogeneity by marking plots unfit for vegetation;

- ability to keep track of changes in genetic structure of phytophage population;

- accounting for the Allee effect $[1,59]$ in the phytophage population;

- universality, applicability to broad class of similar systems.

Peculiarities of theoretical and applied problems related to the biological control of common ragweed stipulate the need for studying the evolution of genetic traits in a population of phytophage. Regarding the evolving ability to fly in Zygogramma suturalis, we assume that the flying ability is recessive and governed by a single diallelic locus with a flight allele $f$ and a flightless (walking) allele $w$. Further assuming that the flight allele inherited in an autosomal manner, the ragweed leaf beetle population consists of the flightless genotypes $w w$ and $f w$, and the flying genotype $f f$. Such hypotheses do not contradict to current knowledge and observations of Zygogramma suturalis, whereas in some other species the flightless phenotype is known to be caused by a single recessive mutation (e.g., in the artificially selected flightless strain of harlequin ladybird Harmonia axyridis [10,61]). However we intentionally consider the recessive flight allele in order to demonstrate that the flight ability might effectively evolve even in such a restricted 'extreme' case. 
Being autonomous the model ignores any external forces that could affect the intrinsic dynamics of the system, and therefore seasonal variations in environmental conditions are not taken into account. Thus the full yearly period of the model corresponds actually to the growing season (mid April - mid October) when both common ragweed and leaf beetle are active. All modelled processes are supposed to be continuous and acting simultaneously.

\subsection{Model description}

Taking into account both demography and genetics, and assuming that flight ability affects the movement of the phytophage genotypes within a field territory $\Omega$ occupied by the ecosystem, we build a model based on a taxis-diffusion-reaction system of PDEs:

$$
\begin{aligned}
& \frac{\partial R}{\partial t}=R\left(r_{R}(\mathbf{x})-c_{R} R\right)-\left(N_{f f}+N_{f w}+N_{w w}\right) \cdot \frac{a R}{1+a h R}+\delta_{R} \Delta R \\
& \frac{\partial N_{f f}}{\partial t}=\frac{a R}{1+a h R} \cdot \frac{1}{N+A} \cdot f_{f f}\left(N_{f f}, N_{f w}, N_{w w}\right)-\mu_{f f} N_{f f}-\operatorname{div}\left(N_{f f} \nabla S_{f f}\right)+\delta_{f f} \Delta N_{f f} \\
& \frac{\partial N_{f w}}{\partial t}=\frac{a R}{1+a h R} \cdot \frac{1}{N+A} \cdot f_{f w}\left(N_{f f}, N_{f w}, N_{w w}\right)-\mu_{f w} N_{f w}-\operatorname{div}\left(N_{f w} \nabla S_{f w}\right)+\delta_{f w} \Delta N_{f w} \\
& \frac{\partial N_{w w}}{\partial t}=\frac{a R}{1+a h R} \cdot \frac{1}{N+A} \cdot f_{w w}\left(N_{f f}, N_{f w}, N_{w w}\right)-\mu_{w w} N_{w w}-\operatorname{div}\left(N_{w w} \nabla S_{w w}\right)+\delta_{w w} \Delta N_{w w} \\
& \frac{\partial S_{f f}}{\partial t}=\kappa_{f f} R-\eta_{f f} S_{f f}+\delta_{S f f} \Delta S_{f f} \\
& \frac{\partial S_{f w}}{\partial t}=\kappa_{f w} R-\eta_{f w} S_{f w}+\delta_{S f w} \Delta S_{f w} \\
& \frac{\partial S_{w w}}{\partial t}=\kappa_{w w} R-\eta_{w w} S_{w w}+\delta_{S w w} \Delta S_{w w}
\end{aligned}
$$

where $R=R(t, \mathbf{x})$ is the density $\left(\mathrm{kg} \mathrm{m}^{-2}\right)$ of common ragweed at spatial position $\mathbf{x}=(x, y) \in \Omega$ at time $t ; N_{f f}=N_{f f}(\mathbf{x}, t), N_{f w}=N_{f w}(\mathbf{x}, t), N_{w w}=N_{w w}(\mathbf{x}, t)$ are the densities (individuals per $\left.m^{2}\right)$ of the three genotypes of the ragweed leaf beetle; $N=N(\mathbf{x}, t)$ is the total density of the leaf beetle population, $N=N_{f f}+N_{f w}+N_{w w}$.

According to the demogenetic model of V.A. Kostitzin [27-30], reproduction terms in the balance equations of the leaf beetle genotypes (2.2-2.4) have the following forms:

$$
\begin{aligned}
f_{f f}\left(N_{f f}, N_{f w}, N_{w w}\right) & =e_{f f f f} N_{f f}^{2}+\left(e_{f f f w}+e_{f w f f}\right) N_{f f} N_{f w} / 2+e_{f w f w} N_{f w}^{2} / 4 \\
f_{f w}\left(N_{f f}, N_{f w}, N_{w w}\right) & =\left(e_{f f f w}+e_{f w f f}\right) N_{f f} N_{f w} / 2+e_{f w f p} N_{f w}^{2} / 2+ \\
& +\left(e_{f f w w}+e_{w w f f}\right) N_{f f} N_{w w}+\left(e_{f w w w}+e_{w w f w}\right) N_{f w} N_{w w} / 2 \\
f_{w w}\left(N_{f f}, N_{f w}, N_{w w}\right) & =e_{w w w w} N_{w w}^{2}+\left(e_{f w w w}+e_{w w f p}\right) N_{f w} N_{w w} / 2+e_{f w f w} N_{f w}^{2} / 4 .
\end{aligned}
$$

The reproduction functions (2.8-2.10) satisfy the Mendelian inheritance and implicitly assume that each genotype is represented by males and females with a constant (1:1) sex ratio. It is also assumed that fecundity (conversion coefficient) $e_{i j}$ of a couple constituted by male and female having respectively genotypes $i$ and $j,(i, j=f f, f w, w w)$ can differ from fecundity $e_{j i}$ of a couple consisting of male with genotype $j$, and female with genotype $i$. In the case of autosomal inheritance $\left(e_{i j}=e_{j i}\right)(2.8-2.10)$ reduce to:

$$
\begin{aligned}
& f_{f f}\left(N_{f f}, N_{f w}, N_{w w}\right)=e_{f f f f} N_{f f}^{2}+e_{f f f w} N_{f f} N_{f w}+e_{f w f w} N_{f w}^{2} / 4 \\
& f_{f w}\left(N_{f f}, N_{f w}, N_{w w}\right)=e_{f f f w} N_{f f} N_{f w}+e_{f w f w} N_{f w}^{2} / 2+2 e_{f f w w} N_{f f} N_{w w}+e_{f w w w} N_{f w} N_{w w} \\
& f_{w w}\left(N_{f f}, N_{f w}, N_{w w}\right)=e_{w w w w} N_{w w}^{2}+e_{w w f w} N_{f w} N_{w w}+e_{f w f w} N_{f w}^{2} / 4 .
\end{aligned}
$$

The other parameters of the model have obvious meaning: $r_{R}$ is the reproduction coefficient of the common ragweed; $c_{R}$ is the intraspecific competition coefficient of common ragweed. Note that ratio of 
these parameters $K_{R}=r_{R} / c_{R}$ represents the carrying capacity of the weed population. Thus, simple logistic growth is assumed for the common ragweed in (2.1). The weed growth can depend on spatial coordinate, i.e., $r_{R}=r_{R}(\mathbf{x})$, which allows taking into account the spatial heterogeneity of the environment by setting a non-zero value of $r_{R}$ everywhere within the spatial domain $\Omega$ except in plots marked as unfit for vegetation, where $r_{R}(\mathbf{x})=0$. Symbol $\nabla$ represents the gradient; $\Delta$ is the Laplacian. It is assumed that the weed plant disperses diffusively with a diffusion coefficient $\delta_{R}$. Diffusion coefficients $\delta_{f f}$, $\delta_{f w}, \delta_{w w}$ characterise intensities of random undirected movements of the phytophage genotype densities. The phytophage consumes the common ragweed according to the Holling type II functional response $g(R)=a R /(1+a h R)$, where $a$ is the searching efficiency of the consumer, $h$ is the handling time. Parameters $\mu_{f f}, \mu_{f w}, \mu_{w w}$ are the mortality coefficients of the leaf beetle genotypes. Non-zero values of parameter $A$ incorporate the Allee effect into the model, allowing an accounting for the declining of the consumer birth rate at low population density $[1,59,72]$. The Allee parameter $A$ in the consumer equations (2.2-2.4) can be interpreted as the "half-velocity constant" because it corresponds to the value of density $N$ at which the effective conversion coefficient of the leaf beetle population is one-half of its maximum [72].

The advective terms in (2.2-2.4) describe the directed movements of the genotype densities. Thus, variables $S_{f f}=S_{f f}(\mathbf{x}, t), S_{f w}=S_{f w}(\mathbf{x}, t), S_{w w}=S_{w w}(\mathbf{x}, t)$ are the taxis velocity potentials of phytophage genotypes that represent stimuli of tactic movements $[64,66]$. Field observations suggest that local food shortage increases spatial activity of the ragweed leaf beetle $[33,34,36]$. Thus, for the modelled phytophagous insect species, taxis stimuli can be interpreted as potential satiation of individual consumers at every spatial position. The decay coefficients of the taxis stimuli $\eta_{f f}, \eta_{f w}, \eta_{w w}$ in balance equations (2.5-2.7) characterize "memory" effects of the insect feeding (or food testing) system, and can be interpreted as rates of food digestion by the respective consumer genotypes. Alternatively, in a mechanical context, these parameters can be viewed as friction coefficients reducing taxis velocity. Intensity of the directed movements (consumer sensitivity to food shortage) is given by the taxis coefficients $\kappa_{f f}$, $\kappa_{f w}, \kappa_{w w}$. The diffusion coefficients of genotype taxis stimuli $\delta_{S f f}, \delta_{S f w}, \delta_{S w w}$ describe various effects of collective insect movements [17] and can be interpreted as viscosity [66]. Details of the method used for taxis modelling, its justification and advantages are given in the earlier papers $[2,16,55,63,64,66]$.

System (2.1-2.7) with the boundary conditions

$$
\nabla R \cdot \mathbf{n}=\nabla N_{f f} \cdot \mathbf{n}=\nabla N_{f w} \cdot \mathbf{n}=\nabla N_{w w} \cdot \mathbf{n}=\nabla S_{f f} \cdot \mathbf{n}=\nabla S_{f w} \cdot \mathbf{n}=\nabla S_{w w} \cdot \mathbf{n}=0, \quad \mathbf{x} \in \partial \Omega,
$$

where $\mathbf{n}$ is the external normal to the boundary $\partial \Omega$, entirely describes spatio-temporal dynamics of the trophic system "common ragweed - leaf beetle" and the evolution of the genetic structure of the phytophage population within a closed spatial domain. Though zero-flux boundary conditions (2.14) are set to suit mainly numerical convenience, one can minimise boundary effects, at least during the initial period of simulation, by considering the domain $\Omega$ larger than the typical spatial scale at which formation and movement of solitary population wave of the ragweed leaf beetle can be observed in natural conditions $[33,34]$.

Besides through immediate observation of the weed and beetle genotype densities, spatio-temporal dynamics of the allele frequencies

$$
\begin{aligned}
& n_{f}=\left(N_{f f}+N_{f w} / 2\right) /\left(N_{f f}+N_{f w}+N_{w w}\right) ; \\
& n_{p}=\left(N_{w w}+N_{f w} / 2\right) /\left(N_{f f}+N_{f w}+N_{w w}\right),
\end{aligned}
$$

can also be traced in every detail with help of the demogenetic model.

\section{Simulations}

In order to carry out numerical simulations we applied the standard method of lines [56]: the continuous demogenetic model was discretized in space with a regular grid consisting of $m \times k$ nodes along the spatial 
coordinates $(x, y)$, approximating the spatial derivatives with the central differences in each node, and introducing dummy nodes on the boundaries. Thus, with $m=k=150$ the original system of PDEs (2.12.7 ) is approximated by $150 \times 150 \times 7=157500$ ordinary differential equations (ODEs). The obtained system of ODEs with the preset initial conditions was integrated with the Runge-Kutta method of the fifth order with automatic time step selection. The accuracy of calculations was checked on the doubled spatial grid.

\subsection{Simulation setup and parameter values}

With the intention to reproduce the qualitative dynamics of real processes observed during the introduction of the ragweed leaf beetle we chose a set of plausible values for the model parameters. Figure 1A shows a rectangular domain $\Omega=L_{x} \times L_{y}$ with a spatial pattern corresponding to a real map of 'Pelagiada' farm (the Stavropol Territory), $L_{x}=4000 \mathrm{~m}, L_{y}=3000 \mathrm{~m}$. It is supposed that initially, the area is heavily infested, with a ragweed density of $R(0, \mathbf{x})=1 \mathrm{~kg} \mathrm{~m}^{-2}$ in all plots suitable for vegetation. Such high density is close to the carrying capacity of the common ragweed $K_{R}=1.17 \mathrm{~kg} \mathrm{~m}^{-2}$ provided by parameters $c_{R}=0.01 \mathrm{~m}^{2} \mathrm{~d}^{-1} \mathrm{~kg}^{-1}$ and $r_{R}=0.0117 \mathrm{~d}^{-1}$. Such value of the Malthusian coefficient $r_{R}$ corresponds to relatively slow increase of the ragweed phytomass (doubling in about two weeks), which is typical for this 'explerent' species visibly lagging in growth at the beginning of the growing season in comparison with native plants $[14,33]$. At the same time, the diffusion coefficient $\delta_{R}=0.5 \mathrm{~m}^{2} \mathrm{~d}^{-1}$, reflecting the relatively rapid dispersal of the common ragweed.
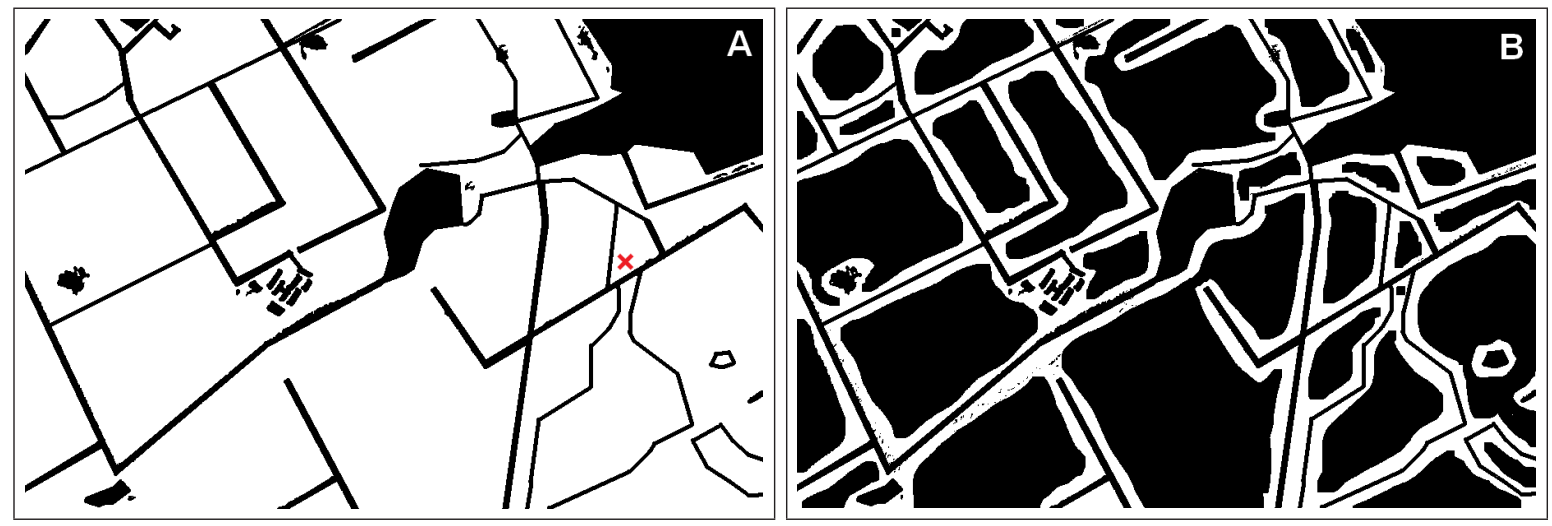

Figure 1. Patterns of spatial domain used in simulations. Black plots are unfit for vegetation (urban areas, roads, forest belts, water bodies, etc.). Map of 'Pelagiada' farm with point of the leaf beetle release merked by cross (A). Hypothetical pattern with strong fragmentation - only roadsides and field edges are fit for the ragweed (B).

As was done in 1978, at the start time, 1500 ragweed leaf beetles are released near the village of Pelagiada (cross-mark in fig. 1A). We assume that all the released beetles were flightless, i.e., initially there were no homozygous individuals $N_{f f}$ and only a small number of heterozygotes $N_{f w}$ (100 beetles) possessing one copy of the flight allele. Thus, according to our assumption, most of the released insects (1400 beetles) were homozygous $N_{w w}$. The initial frequency of the flight allele (2.15) in the whole population was quite low: $n_{f}=1 / 300$.

We also assume that all ecological characteristics of the beetle (fecundity, mortality rates, etc.) are identical for different genotypes except their movement ability, i.e., both taxis and diffusion coefficients of the flying genotype $f f$ are five times higher than the values for the flightless genotypes $f w$ and $w w$ : $\kappa_{f f}=0.025 \mathrm{~m}^{4} \mathrm{~kg}^{-1} \mathrm{~d}^{-2} ; \kappa_{f w}=\kappa_{w w}=0.005 \mathrm{~m}^{4} \mathrm{~kg}^{-1} \mathrm{~d}^{-2} ; \delta_{f f}=15 \mathrm{~m}^{2} \mathrm{~d}^{-1} ; \delta_{f w}=\delta_{w w}=3 \mathrm{~m}^{2} \mathrm{~d}^{-1}$. For 'walkers' we use a field estimation of the diffusion coefficient obtained by Kovalev and Vechernin $[36,37]$. 
The values of taxis coefficients result in realistic taxis velocities in simulations: up to 8 and $40 m d^{-1}$ for 'walkers' and 'fliers' respectively. The values of the other parameters determining spatial behaviour of beetles are equal for all genotypes: $\eta_{f f}=\eta_{f w}=\eta_{w w}=0.01 d^{-1} ; \delta_{S f f}=\delta_{S f w}=\delta_{S w w}=0.05 \mathrm{~m}^{2} \mathrm{~d}^{-1}$.

The ragweed leaf beetles go through their life cycle in one year. Assuming that nearly all (98,5\%) beetles die within this period, we obtain the mean instantaneous mortality rates $\mu_{f f}=\mu_{f w}=\mu_{w w}=$ $0.0114 d^{-1}$. We also take equal values for all conversion coefficients in genotype reproduction functions (2.11-2.13), $e_{i j}=2625 \mathrm{~kg}^{-1}(i, j=f f, f w, w w)$.

Based on data obtained in field and laboratory experiments [5,36,37], we assume that the maximum individual ration of the leaf beetle is $0.028 \mathrm{gd}^{-1}$, which gives us the handling time value $h=35700$ $d \mathrm{~kg}^{-1}$. Taking into account the fact that the ragweed beetle, especially during its larva stage, is not very efficient in local searching $[5,33]$, we set the respective parameter of the functional response to plausibly low value $a=0.6 \times 10^{-4} \mathrm{~m}^{2} \mathrm{~d}^{-1}$.

Finally, according to the modelling hypothesis, the Allee effect influences the recruitment of the ragweed leaf beetle with a small value of the parameter $A=0.001$.

Thus, some of the model parameters have been identified based on general knowledge of the system, or on field observations and laboratory experiments. There are, however, coefficients with unknown values which are hard to identify, like the conversion coefficients of genotypes, or the Allee parameter. Such parameters are set to reasonably arbitrary values resulting in realistic dynamics of the model. Though the exact values of some coefficients are not available, we observed no significant qualitative changes in the results upon moderately varying the parameter values in additional test simulations.

\subsection{Simulation results}

Running the model with the given parameter values shows that system development comprises four clearly distinguishable phases:

- An initial phase of phytophage population outbreak, lasting for about one-two years during which the initial patch of the beetle population density gradually increases in size, causing local suppression of the ragweed;

- Formation and onward movement of the phytophage population wave, effectively suppressing the ragweed and expansion of the territory occupied by the leaf beetle (see fig. 2). The wave is characterised by very high density of phytophages in the wave front (up to 2000 individuals per $m^{2}$ and more in our simulation);

- Rapid transformation of the genetic structure of the invading phytophage population, supplanting of virtually all walking beetles by 'flyers' within only 4 years (see figs 3,4 );

- Stabilization of spatially heterogeneous wave regime with irregular appearance of isolated nidi of common ragweed being promptly suppressed by subsequent aggregations of phytophage. The spatially averaged density of the ragweed does not exceed $100 \mathrm{~g} \mathrm{~m}^{-2}$, which is less than $10 \%$ of the ragweed carrying capacity (see fig. 5), while local maximums can intermittently reach $K_{R}$.

As it was stressed earlier, though the stabilization phase is an important characteristic of the system dynamics in general and, particularly of the phytophage's ability to ensure a successful biological control, maintaining the weed biomass below some acceptable threshold [55], one should bear in mind that this phase of the model run is strongly affected by the zero-flux boundary conditions. Thus, the long-term computations are mainly of theoretical interest. Nevertheless, apart from studying a transitive process, it is worth drawing attention to peculiarities in the system, that are revealed by the long-term simulations.

First of all, one can see that, at least under the model assumptions, the evolution of the flight ability is not unidirectional. The time-plot in the right pane of fig. 5 shows that the frequency of the flight allele in the phytophage population fluctuates even though the system approaches stabilization. Our observations suggest that flightless beetles (those with the lower values of both diffusion and taxis coefficients) can have an advantage over the flying individuals during relatively short starvation periods due to a largescale decrease of the weed density, i.e., when an increasing migration intensity of the consumer cannot improve its fitness because an acute food shortage is everywhere. 


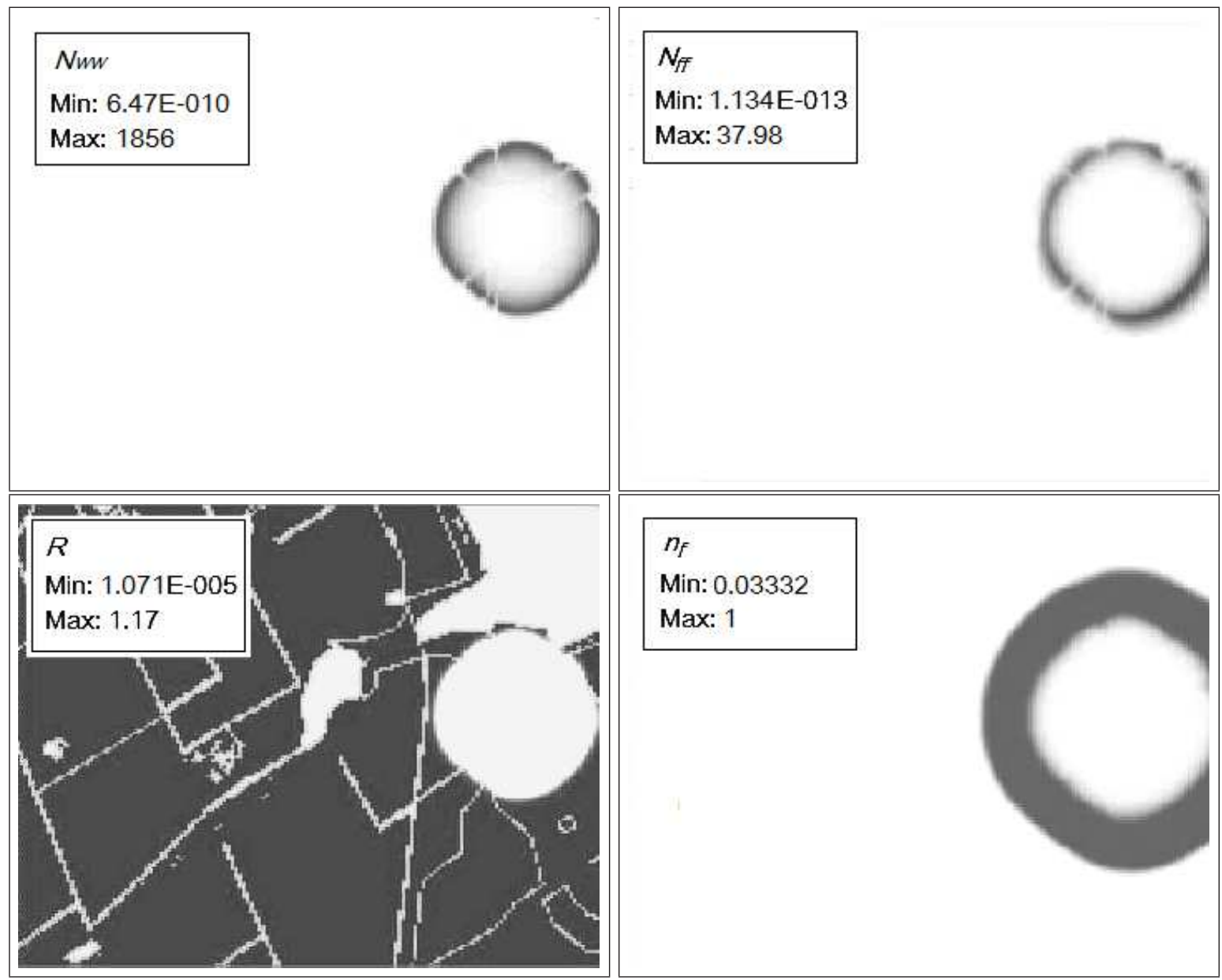

FiguRE 2. Snapshot of spatial distribution of the model variables after two simulated years $(t=2 y r)$. Though most of the leaf beetles are flightless $(95.5 \%)$ the fraction of the flying beetles gradually increases. Note that wave of the flight allele frequency $n_{f}$ passes ahead of the front of the population wave of the flying genotype $f f$.

However, ultimately almost the whole population is composed of beetles having the $f f$ genotype .

\subsection{Additional numerical experiments}

In order to elucidate the factors accelerating the evolution in the model, additional simulations with $A=0$ have been performed. It is detected that in the absence of the Allee effect, the transformation of the genetic structure towards the domination of flying individuals becomes negligibly slow and does not have any noticeable impact on the system.

A numerical experiment with a homogeneous initial distribution of the introduced phytophages has confirmed that the recommendation to concentrate the release of biocontrol agents [33-37] is highly practical and important in provoking the outbreak of phytophagous insects. Violation of this condition does not allow to exploit the advantages of a phytophage's solitary population waves in suppressing the ragweed. In the absence of the wave, the flight ability does not evolve either.

Another important aspect that can influence the effectiveness of the biological method of weed control is primary productivity of the trophic system, that supplies the energy needed for the upper trophic 

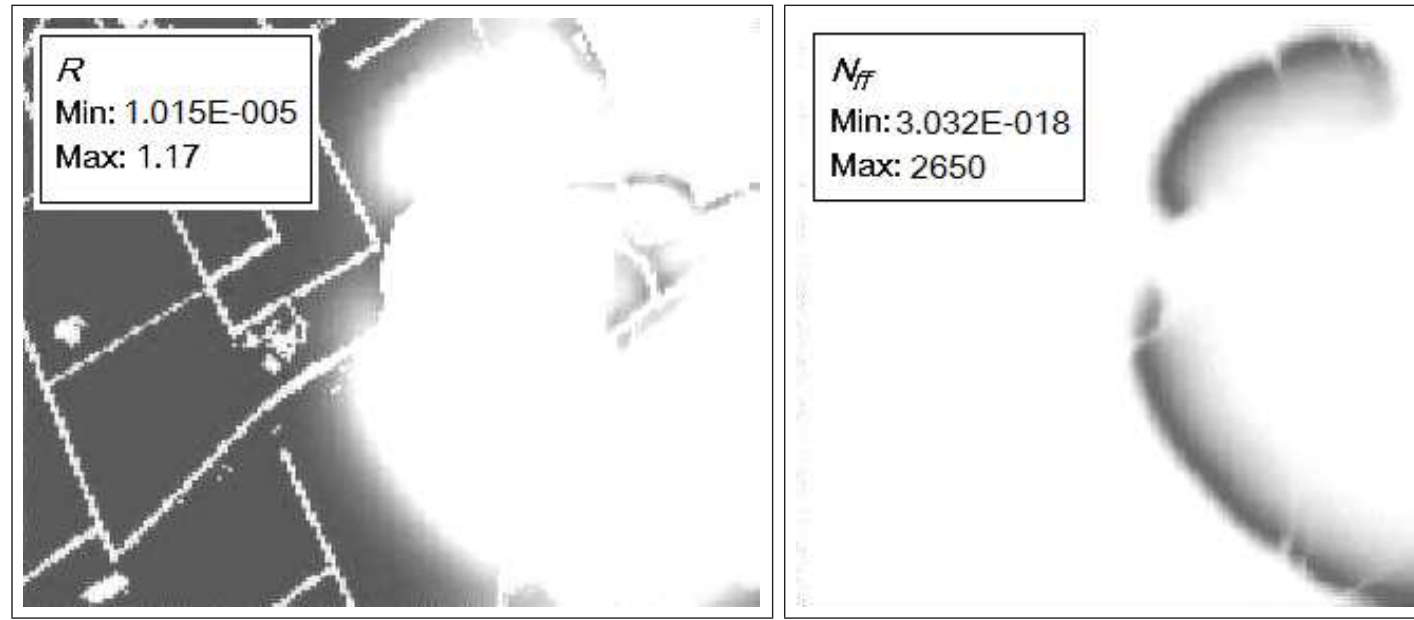

FiguRE 3. Spatial distribution of common ragweed (left pane) and of the flying genotype of the ragweed leaf beetle (right pane) after four simulated years $(t=4 \mathrm{yr})$.
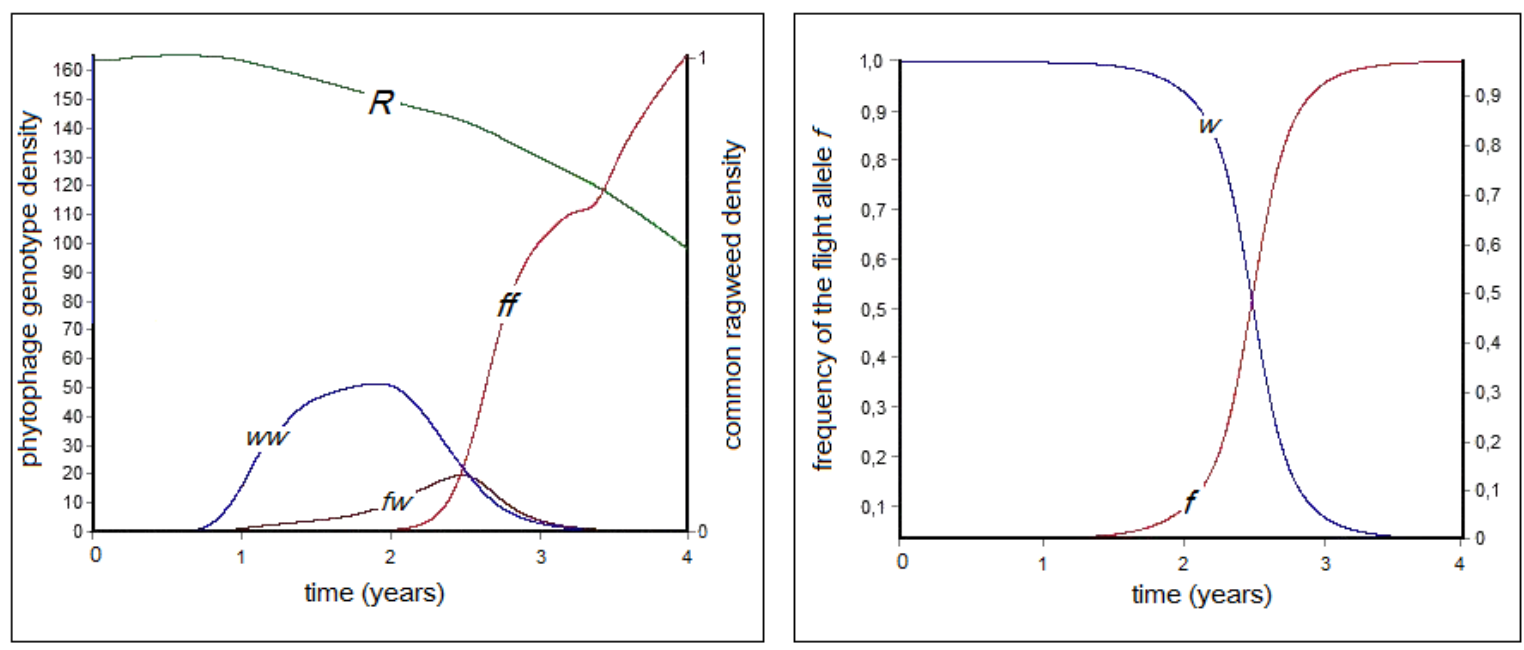

FiguRE 4. Simulated dynamics of the spatially averaged densities of common ragweed $(R)$ and three genotypes of the ragweed leaf beetle (left pane), and evolution of the flight $(f)$ and flightless $(w)$ allele frequencies (right pane).

levels. The effective use of the phytophagous biocontrol agent requires the creation of special conditions, in particular, release areas with high weed density [33-37]. Figure 6 shows how the increase of the parameter $c_{R}$ (i.e., diminishing the ragweed carrying capacity) affects both the establishment of the leaf beetle population and the development of its flight ability. Gradually increasing $c_{R}$, we performed simulations for the 10-year period, starting each run with equal proportions of 'flyers' and 'walkers' $\left(n_{f}=0.5\right)$. The results suggest that the lower the primary productivity of the ragweed, the lower the relative effect of the introduction of the phytophage into the ecosystem. The model predicts that with high value of $c_{R}$ (e.g., $c_{R}>0.05$ ), despite of the successful establishment of phytophage, its biocontrol efficiency is weak, the population wave is not well-shaped, slow moving and does not suppress the weed effectively. Extremely poor level of weed supply (taking a coefficient of intraspecific competition $c_{R}>0.08$ $\mathrm{m}^{2} \mathrm{~d}^{-1} \mathrm{~kg}^{-1}$ ) could not support neither acclimatization of the phytophage, nor the formation of SPW, 

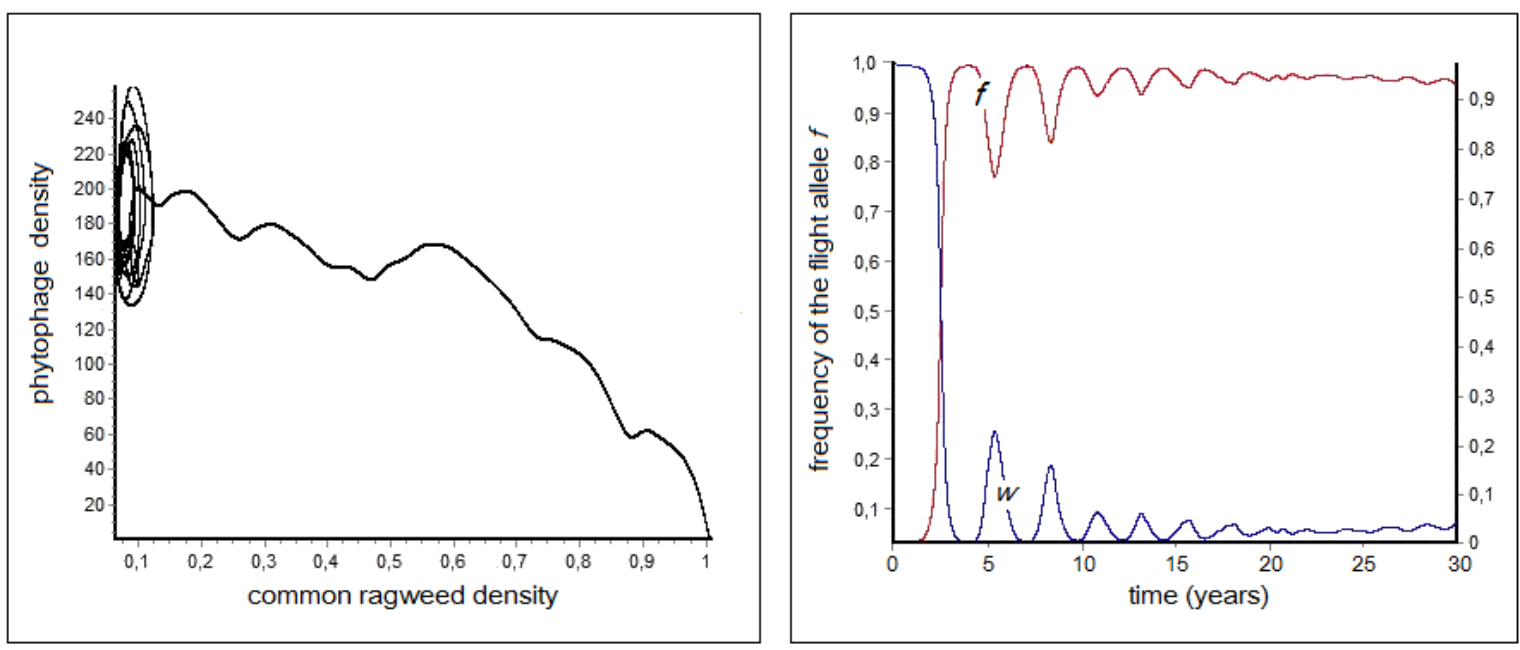

Figure 5. Phase trajectories of the model presented on the plane of the spatially averaged population densities (left pane), and long-term evolution of the allele frequencies (right pane).

nor the suppression of the ragweed. Moreover, a too strong lowering of the weed production (e.g., caused by excessive herbicide application) drives the population of introduced phytophages to extinction.

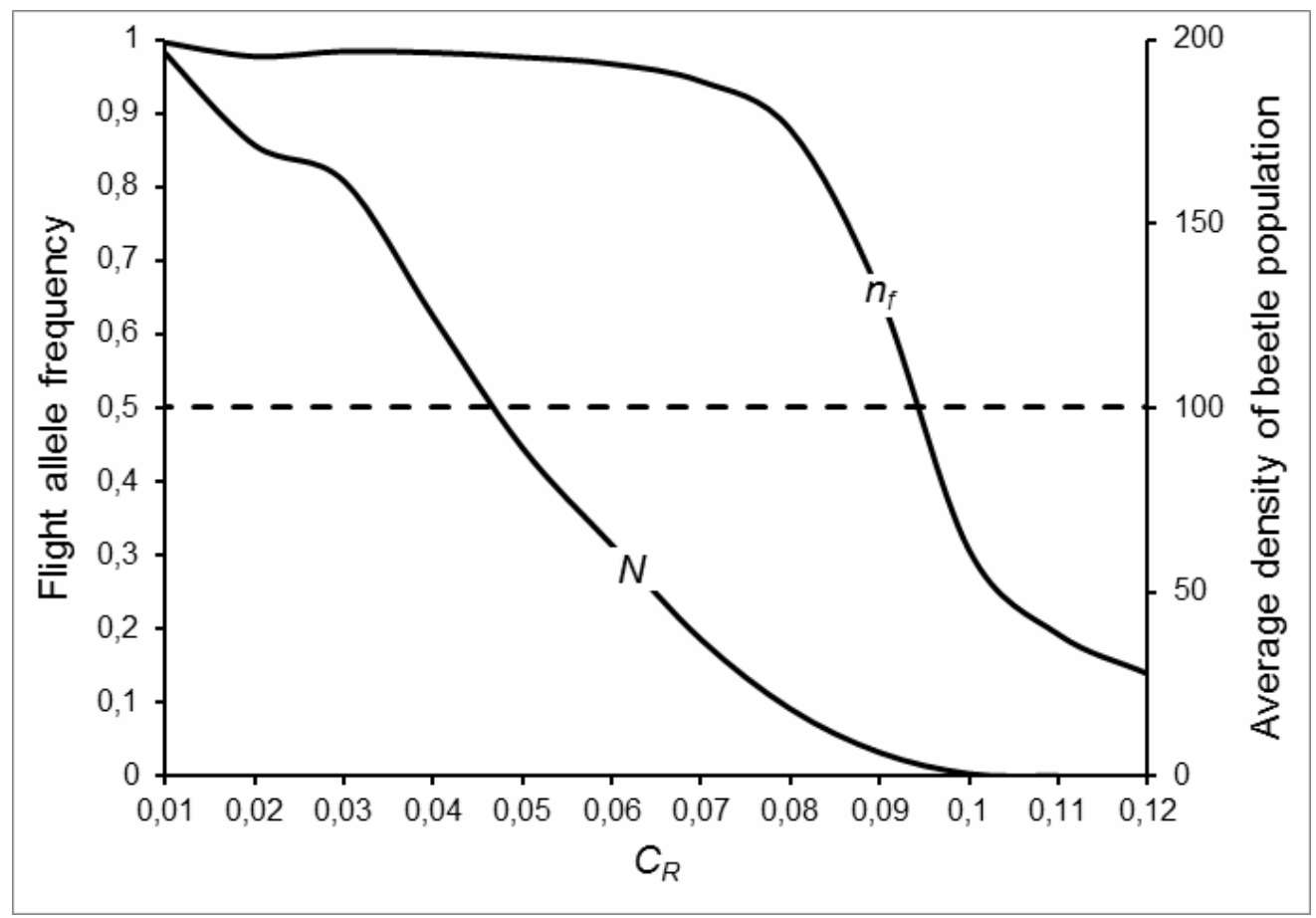

Figure 6. Spatially averaged density $(N)$ and flight allele frequency $\left(n_{f}\right)$ of the leaf beetle population after ten simulated years, depending on the intraspecific competition coefficient of the ragweed. 
Interestingly, tendency of evolving toward increasing frequency of flight allele in the phytophage populations is kept even with rather high values of $c_{R}$, although the evolution is slowed down. Therefore, this result does not really explain why the flying allele of the ragweed leaf beetle is evolutionary disadvantageous in its native habitat. The explanation is suggested by another supplementary experiment where we were varying fragmentation of spatial domain rather than values of the model parameters. It turned out that use of spatial pattern with more plots unfit for common ragweed (fig. 1B) increases chances of non-flying beetles to win the war of evolution (not shown). However, this 'backward' transformation requires much longer time (about 40 and 80 years in our simulations with recessive and dominant 'flight' allele respectively) than flight development of the ragweed beetle observed upon its introduction in the South of Russia because now evolutionary process is not amplified by action of solitary population waves.

\section{Discussion}

The model $(2.1-2.7,2.14)$ is built with the use of a number of hypotheses simplifying the real situation. In particular, the model ignores the stage transformations of the phytophage, seasonal changes of environmental factors, passive transport of beetles with wind, random events of long-distant dispersal of weed seeds and insects, appearance and coalescence of secondary foci that accelerate the phytophage dispersal, soil disturbances, the presence of other species in the ecosystem, and other factors. Nevertheless, the results presented here are in good qualitative agreement with the field observations and earlier theoretical studies of the ragweed leaf beetle introduction $[32,34,37]$. The model easily reproduces the observed patterns of SPW motion [34], including the so-called 'edge effect' consisting in a relatively higher density of weed in the borders of a field behind the passing wave of phytophage (see secondary emerging patches of the ragweed in fig. 3 ).

In addition to the common ragweed and the ragweed leaf beetle, our model can also describe the dynamics of other species, such as cultural plants competing with the ragweed, and entomophagous predator of the leaf beetle. We however, chose not to present these results here. They will be considered in separate papers. It should be emphasized that, even in the extended trophic systems, the basic relationships between weed and phytophage populations play the key role in determining the overall dynamics and success of biological methods of control, including the so-called phyto-cenological method.

The generic quality of the model allows its application to other known or forthcoming campaigns of biocontrol agent acclimatization $[21,24,42,52,53,71]$ as well as to various cases of species invasions into particular habitats $[10,47]$.

The model is based on a taxis-diffusion-reaction system that was completely studied earlier [55, 62 , 66]. Combining this approach to explicit modelling of animal spatial behaviour $[2,16,55,62,66]$ with demogenetic equations of V.A.Kostitzin [27-30] provides a natural way to directly describe the spatiotemporal dynamics of a genetically structured population in terms of genotype densities [67,68]. With the right parameter values, our model allows the simulation of a continuous transitions between the two dynamic regimes corresponding to the 'extreme' parameter sets that differ trough the coefficients of taxis and diffusion used for the flying and flightless phenotypes. The model allows the tracing of genetic transitions that occur depending on the current state of the constantly changing system. The resulting spatial behaviour of insects of each genotype entirely determines the genotype's fitness. Thus, the model, in particular, can be used to investigate which conditions are favourable for the alternative strategy of the biocontrol agent known as "search-and-destroy" and "lying-in-wait" [44]. Any parameter, not only diffusion and taxis coefficients can be varied to distinguish one genotype from another. This allows to take into account the fitness cost of an advantageous mutation.

For the sake of simplicity in our study, we assume that the flying ability of the $f f$ genotype neither reduce its conversion coefficient nor increase its mortality rate. This assumption is supported by the fact that in general, insects spend more energy for walking than for flying [4,57]. Moreover, according to field observations even flying imago of Zygogramma suturalis migrate by flight rarely, mainly during the autumn period of mating $[5,32,34]$. That is why values of taxis and diffusion coefficients for 'fliers' are only moderately (five times) exceed values used for 'walkers'. Regarding the ability of phytophages to achieve 
sustainable biological control of the weed, the interplay between trophotaxis and diffusion coefficients (see [55]) allows all genotypes to suppress ragweed in simulations with given parameter values. Under these circumstances, numerical experiments demonstrate that extremely high population density within the wave front promotes strong natural selection in favour of beetles with a higher migration activity. The flight ability evolves very fast. After formation of the phytophage wave, it takes no more than four years (about six generations) to replace almost all 'walkers' with 'flyers'.

It is important that field observations of the evolutionary changes that were noticed in the eighties, have also been corroborated by morphological analyses. Kovalev and Brodsky $[3,32]$ have demonstrated that flying imagos of Zygogramma suturalis collected in the Stavropol Territory differ morphologically from specimens collected in Canada and the USA by the structure of the axillary apparatus, including the development of wing muscles and axillary sclerites.

The additional numerical experiments, in particular, computations with vastly fragmented hypothetical spatial pattern (fig. 1B) suggest that in the past, environmental heterogeneity might have played an important role in evolutionary loss of the flight ability of Zygogramma suturalis. Indeed, according to the modern views, long before the beginning of the worldwide invasion by common ragweed, caused by its anthropogenic dissemination, the natural habitats of Ambrosia artemisiifolia were isolated from phytocenoses, and limited to rather small disconnected areas in the North-West coast of the modern Florida peninsula [32]. Being a narrow oligophage, the ragweed leaf beetle did not have to leave patches occupied by its host plant. Thus, individuals with weakened wings had clear evolutionary advantages over those migrating far away from the rare sources of their food [32]. But our simulations answer another question: Why didn't the ragweed leaf beetle evolve the flying ability on the American continent nowadays, when the common ragweed occupies huge territories due to human activity? — Simply because spreading of the leaf beetle over the American continent was not accompanied by solitary population waves. Formation of SPW is never possible in the conditions of a species' native habitat, where numerous competitors and missing vacant econiche prevent an outbreak of the phytophage population $[34,36,37]$.

Our simulation results confirm the earlier hypothesis that the formation of the phytophage population wave is an important factor in amplifying the efficiency of a weed biocontrol agent. By varying the values of parameters and changing simulation conditions, the model helps to understand the roles played by the Allee effect, primary production, and initial heterogeneity.

Additional simulations confirm the fundamental importance of the Allee effect $[1,59]$ for obtaining realistic model prediction. This is not very surprising because extinction of maladapted genotypes should always accelerate the evolution, forcing the natural selection of a genetype with higher fitness. In particular, our results are in line with recent conclusions about the crucial role that the Allee effect can play in the wave front of an expanding population, promoting diversity in travelling waves of colonisation $[9,54]$, and enhancing the adaptation towards a range expansion phenotype $[18,39]$. In a more general context, studying the wave patterns of expanding populations is of great theoretical interest and can be applied to the broad range of problems from evolution of biodiversity to the consciousness evolution $[31,38]$.

What can be a rationale for inclusion of the Allee effect into the population model of the ragweed leaf beetle? In our opinion the Allee effect in Zygogramma suturalis can be caused by demographic stochasticity at low density [13] coupled with sexual dimorphism in body size and assortative mating for body size [58]. These factors make the ragweed beetle liable to the mate-finding Allee effect, that often plays a crucial role in ecosystem invasions and biological control [6].

\section{Conclusion}

We share the opinion that the principal peculiarity of weed biological control, that distinguishes it from biocontrol of insect pest consists in the fact that the successful application of a phytophagous agent is impossible without the formation of phytophage SPW effectively suppressing the weed [34]. Ignoring or incomprehension of this principle may lead to a wrong estimation of the biocontrol agent efficiency. Moreover, we are convinced that any reasoning about 'phytophage efficiency' without considering conditions of SPW formation is just meaningless. Not phytophage, but the method of its application can 
be either efficient or inefficient, and elaboration of an efficient method of weed control should pursue the creation of appropriate conditions for a phytophage wave formation under existing circumstances. What are these conditions?

First of all, it has been particularly emphasised that the formation of the phytophage SPW is impossible in the home-range conditions of species [34,36,37]. Both field experiments and results of mathematical modelling confirm the importance of the following two requirement which allow the initial increase of the biocontrol agent population namely a concentrated mode of phytophage introduction, and a ragweed density as high as possible (at least 1000 germs per $m^{2}$ ) at the release plot. Thus, in order to provoke the formation of an SPW of Z. suturalis in 'Pelagiada' farm, the soil in the primary release plot ( $\approx 2$ ha) was intensively ploughed prior to release, having achieved the exceptionally high density of common ragweed - $1 \mathrm{~kg}$, or 10000-12000 young plants per $m^{2}$ [33,34]. Note that in spite of very high level of ragweed infestation, ignoring of this preliminary works stage has caused lowering the effect of the ragweed control measures in former Yugoslavia [22].

A little quantity, 1500 individuals of the ragweed leaf beetles released in 1978 in the Stavropol Territory turned into a very large population consisting of millions insects. With expansion of the phytophage SPW, the weedless area increased as follows: $1979-1$ ha; $1980-4$ ha; $1981-200$ ha; $1982-600$ ha; 1983 - 5500 ha; $1984-20000$ ha; $1986-300000$ ha $[33,36,37]$. This resulted in increasing the yield of agricultural crops by 2-3.5 times (see details in [36]). Field samples taken in 'Pelagiada' farm showed that the seed bank of common ragweed has been reduced from 24000 seeds per $m^{2}$ in 1980 to 35 seeds per $m^{2}$ in $1985[33,36]$ Quite effective results have been achieved in the fields of crop rotation: Until now the common ragweed can be found mainly in the border of the fields [35]. Effective results were also obtained with SPW of the leaf beetle in Georgia [33].

Additionally, the established ragweed leaf beetle conditioned the subsequent acclimatization of the predacious stink bug (Perillus bioculatus F.) in the North Caucasus, having become a food resource of this useful insect species [23]. This entomophage is the most promising biocontrol agent against the Colorado potato beetle (Leptinotarsa decemlineata Say), and its establishment is of great economic significance for European growers of vegetables [60].

Unfortunately after the dissolution of the Soviet Union, the extensive large-scale studies, related to biological control of the common ragweed has been phased out. The expanding wave fronts of the ragweed leaf beetle continued spreading in the absence of observations. Eventually, however, this process resulted in a number of important qualitative changes in phyto- and agrocenoses of the South of Russia including considerable (by an order of magnitude) decline of the soil seed bank and the density of the ragweed plants, and restoration of natural succession previously arrested by the common ragweed [37].

Acclimatization of Zygogramma suturalis in the former USSR is listed among the other widely known successful weed control projects that resulted in spectacular declines in the abundance of the target plants $[15,19,49]$. This makes all the more surprising the recent superficial interpretation of the role of the ragweed leaf beetle by the authors of [51], who analyse the current (i.e., twenty years after complete termination of the field works) distribution of the phytophage and make conclusion about its "negligible efficiency". On one hand this conclusion is not well-grounded because the current distribution of the ragweed beetles has nothing to do with the method of its application. In the American continent, $Z$. suturalis never creates dense aggregations, it is just the normal behaviour of this species [33, 34,36]. Moreover, one should not even expect the phytophage to exterminate its host plant in natural conditions $[69,70]$. The biomethod consists in creating artificial conditions for formation of a phytophage SPW, and the method has already demonstrated conclusive evidence of its efficiency. On the other hand, this misunderstanding raises a problem of the unclearly defined meaning of 'successful biological control' varying between different publications. In their recent polemic paper, discussing the possible criteria for successful weed control, Hoffmann and Moran suggest asking the crucial question: "What would the situation have been without any biological control?" [20]. This criterion may help the authors of [51] to reconsider their evaluation. At the same time, dissemination of erroneous assessments may be harmful to the theory and the application of weed control. In particular, confused researchers may hesitate about 
the introduction of useful insect species that has already been tested for specificity $[15,19,33]$, instead considering export to Europe of the polyphagous leaf beetle Ophraella communa LeSage, potentially dangerous for agriculture (see, e.g., [26]). The authors of another curious paper [12] study the feeding behaviour of Zygogramma suturalis, simulating the leaf beetle attacks by means of mechanical perforation. Why not work with real beetles in natural conditions? Their impact on the ragweed is far from limited to mechanical damage.

In parallel with $Z$. suturalis being used for substantial reduction of the huge biomass of $A$. artemisiifolia, in the present stage of the ragweed control, acclimatization of pollen- and seed-feeders (Trigonorhinus tomentosus and Euaresta bella), that might turn out efficient in suburban territories where the ragweed leaf beetles cannot form SPW, seems to be a promising affair [14]. Complementing field work, mathematical models can help unravelling the role of various factors affecting the dynamics of these biocontrol agents.

Acknowledgements. The authors thank two anonymous referees for highly valuable advices and useful comments that helped improving the presentation of our results. We are thankful to Dr. Marc Lucas for his kind help with English-language editing of the manuscript. We also thank Dr. Inna Senina for detailed discussion of the theoretical approach. The work was partially supported by Federal Target Programs "Research and Pedagogical Cadres for Innovative Russia", for 2009-2013. Project: New approach to consistent biological control of common ragweed and Colorado potato beetle: field studies, mathematical modelling, and practical recommendations, 1.07.2012-15.11.2013 (project code 2012-1.1-12-000-1001-033, grant agr. 8044), and by the internal grants of the Southern Federal University.

\section{References}

[1] W.C. Allee. Animal aggregations: a study in general sociology. Chicago Univ. Press, Chicago, 1931.

[2] R. Arditi, Yu. Tyutyunov, A. Morgulis, V. Govorukhin, I. Senina. Directed movement of predators and the emergence of density-dependence in predator-prey models. Theor. Popul. Biol., 59 (2001), No. 3, 207-221.

[3] A.K. Brodsky. Structure, functioning and evolution of the insect wing articulation. Lectures on the XVI Annual Readings in Memory of N.A. Kholodkovsky (1 April, 1988). Nauka, Leningrad, 1989, 3-47.

[4] A.K. Brodsky. The evolution of insect flight. Oxford University Press, Oxford, New York, Tokio, 1994.

[5] V.N. Cherkashin (1985) Acclimatization of the ragweed leaf beetle Zygogramma Suturalis Fabr. (Coleoptera, Chrysomelidae) in Stavropol Krai and possibilities of its use for control of common ragweed. Summary of PhD thesis (06.01.11plant protection). Georgian Research Institute of Plant Protection, Tbilisi, 1985, 24 pp. [in Russian]

[6] F. Courchamp, T. Clutton-Brock, B. Grenfell. Inverse density dependence and the Allee effect. Trends Ecol. Evol., 14 (1999), No. 10, 405-410.

[7] T. Czárán. Spatiotemporal models of population and community dynamics. Chapman and Hall, London, 1998.

[8] L. Edelstein-Keshet. Mathematical models in biology. McGraw-Hill, New York, 1988.

[9] C.A. Edmonds, A.S. Lillie, L.L. Cavalli-Sforza. Mutations arising in the wave front of an expanding population. Proc. Nat. Acad. Sci. USA, 101 (2004), 975-979.

[10] B. Facon, L. Crespin, A. Loiseau, E. Lombaert, A. Magro, A. Estoup. Can things get worse when an invasive species hybridizes? The harlequin ladybird Harmonia axyridis in France as a case study. Evol. Appl., 4 (2011), 71-88.

[11] W.F. Fagan, M.A. Lewis, M.G. Neubert, P. Van Den Driessche. Invasion theory and biological control. Ecol. Lett., 5 (2002), No. 1, 148-157.

[12] B. Gard, F. Bretagnolle, F. Dessaint, B. Laitung. Invasive and native populations of common ragweed exhibit strong tolerance to foliar damage. Basic Appl. Ecol., 14 (2013), 28-35.

[13] J. Gascoigne, L. Berec, S. Gregory, F. Courchamp. Dangerously few liaisons: a review of mate-finding Allee effects. Popul. Ecol., 51 (2009), No. 3, 355-372.

[14] E. Gerber, U. Schaffner, A. Gassmann, H.L. Hinz, M., Seier, H. Müller-Schärer. Prospects for biological control of Ambrosia artemisiifolia in Europe: learning from the past. Weed Res., 51 (2011), 559-573.

[15] R.D. Goeden, L.A. Andres. Three recent successes outside of North America. in Handbook of Biological Control (T.S. Bellows, T.W. Fisher, Eds.). Academic Press, San Diego, CA, USA, 1999, 884-885.

[16] V.N. Govorukhin, A.B. Morgulis, Y.V. Tyutyunov. Slow taxis in a predator-prey model. Dokl. Math., 61 (2000), No. $3,420-422$.

[17] D. Grünbaum. Using spatially explicit model to characterize foraging performance in heterogeneous landscape. Am. Nat., 151 (1998), No. 2, 97-115.

[18] O. Hallatschek, D.R. Nelson. Gene surfing in expanding populations. Theor. Popul. Biol., 73 (2008), 158-170.

[19] P. Harris. Classical biocontrol of weeds: Its definitions, selection of effective agents, and administrative-political problems. Can. Entomol., 123 (1991), 827-849. 
[20] J.H. Hoffmann, V.C. Moran. Assigning success in biological weed control: what do we really mean? in Proceedings of the XII International Symposium on Biological Control of Weeds (M.H. Julien, R. Sforza, M.C. Bon, H.C. Evans, P.E. Hatcher, H.L. Hinz, B.G. Rector, Eds.), CABI, Wallingford, UK, 2008, 687-692.

[21] C.B. Huffaker. A comparison of the status of biological control of St. John's wort in California and Australia. Mushi, 39 (1967), No. suppl., 51-73.

[22] J. Igrc, J.C. DeLoach, V. Žlof. Release and establishment of Zygogramma suturalis F. (Coleoptera: Chrysomelidae L.). Biol. Control, 5 (1995), No. 2, 203-208.

[23] V.Y. Ismailov, I.S. Agas'eva. Predaceous stink bug Perillus bioculatus Fabr. A novel view on possibility of acclimatization and perspectives of use. Zashchita i karantin rasteniy, 2 (2010), 30-31. [in Russian]

[24] M.N. Julien, M.W. Griffiths. Biological control of weeds: a world catalogue of agents and their target weeds, 4th edn. CABI Publishing, Wallingford, UK, 1998.

[25] E.F. Keller, L.A. Segel. Initiation of slide mold aggregation viewed as an instability. J. Theor. Biol., 26 (1970), $399-415$.

[26] L. Kiss. Is Puccinia xanthii a suitable biological control agent of Ambrosia artemisiifolia? Biocontrol Sci. Techn., 17 (2007), No. 5, 535-539.

[27] V.A. Kostitzin. Biologie mathématique. Paris, Librairie Armand Colin. 1937.

[28] V.A. Kostitzin. Equations diffèrentielles générales du problème de sélection naturelle. C. R. Acad. Sci, 206 (1938), $570-572$

[29] V.A. Kostitzin. Sur les coefficients mendeliens d’hérédité. C. R. Acad. Sci, 206 (1938), 883-885.

[30] V.A. Kostitzin. Sur les équations diffèrentielles du problème de la sélection mendélienne. C. R. Acad. Sci, 203 (1936), 156-157.

[31] O.V. Kovalev. A universal model of the biosphere evolution and the consciousness evolution. International Symposium "Ecosystem Evolution". Paleontological Institute of the Russian Academy of Sciences, Moscow, 1995, p. 47.

[32] O.V. Kovalev. Microevolutioal processes in population of Zygogramma suturalis F. (Coleoptera, Chrisomelidae) introduced from Nort America to the USSR. in: Theoretical Principles of Biological Control of the Common Ragweed (O.V. Kovalev, S.A. Belokobylsky, Eds.). Proceedings of the Zoological Institute. vol. 189. "Nauka" Publishing House, Leningrad Branch, Leningrad, 1989, 139-165. [in Russian]

[33] O.V. Kovalev. Spread of adventive plants of Ambrosieae tribe in Eurasia and methods of bilogical control of Ambrosia L. (Asteraceae). in: Theoretical Principles of Biological Control of the Common Ragweed (O.V. Kovalev, S.A. Belokobylsky, Eds.). Proceedings of the Zoological Institute. vol. 189. "Nauka" Publishing House, Leningrad Branch, Leningrad, 1989, 7-23. [in Russian]

[34] O.V. Kovalev. The solitary population wave, a physical phenomenon accompanying the introduction of a chrysomelid. in: New Developments in the Biology of Chrysomelidae. (P. Jolivet, Ed.) SPB Academic Publishing bv, The Hague, The Netherlands, 2004, 91-601.

[35] O.V. Kovalev, Yu.V. Tyutyunov, L.P. Iljina, S.V. Berdnikov. On the efficacy of introduction of American insectsphytophages of common ragweed (Ambrosia artemisiifolia L.) in the South of Russia. Entomological Review, 92 (2013), 251-264. [in Russian].

[36] O.V. Kovalev, V.V. Vechernin. Description of a new wave process in population with reference to introduction and spread of the leaf beetle Zygogramma suturalis F. (Coleoptera, Chrysomelidae). Entomological Review, 65 (1986), 93-112.

[37] O.V. Kovalev, V.V. Vechernin. Discovering and description of the phenomenon of formation of solitary population wave of introduced insects. in: Theoretical Principles of Biological Control of the Common Ragweed (O.V. Kovalev, S.A. Belokobylsky, Eds.). Proceedings of the Zoological Institute. vol. 189. "Nauka" Publishing House, Leningrad Branch, Leningrad, 1989, 105-120. [in Russian]

[38] O.V. Kovalev, S.G. Zhilin. (Eds.) Phase transition in biological systems and the evolution of biodiversity. Nuclear Physics Institute Publishing House, St. Petesburg, 2007. [in Russian]

[39] R. Lehe, O. Hallatschek, L. Peliti. The rate of beneficial mutations surfing on the wave of a range expansion. PLoS Comput. Biol., 8 (2012), No. 3, e1002447.

[40] M.A. Lewis. Spatial coupling of plant and herbivore dynamics: the contribution of herbivore dispersal to transient and persistent "waves" of damage. Theor. Popul. Biol., 45 (1994), No. 3, 277-312.

[41] L.N. Medvedev. Variability of Zygogramma suturalis F. population introduced to the USSR. in: Theoretical Principles of Biological Control of the Common Ragweed (O.V. Kovalev, S.A. Belokobylsky, Eds.). Proceedings of the Zoological Institute. vol. 189. "Nauka" Publishing House, Leningrad Branch, Leningrad, 1989, 177-181. [in Russian]

[42] P.J. Moran, C.J. DeLoach, T.L. Dudley, J. Sanabria. Open field host selection and behavior by tamarisk beetles (Diorhabda spp.) (Coleoptera: Chrysomelidae) in biological control of exotic saltcedars (Tamarix spp.) and risks to non-target athel (T. aphylla) and native Frankenia spp. Biol. Control, 50 (2009), 243-261.

[43] A. Morozov, S. Petrovskii. Excitable population dynamics, biological control failure, and spatiotemporal pattern formation in a model ecosystem. B. Math. Biol., 71 (2009), 863-887.

[44] W.W. Murdoch, J. Chesson, P.L. Chesson. Biological control in theory and practice. Am. Nat., 125 (1985), No. 3, 344-366.

[45] J.D. Murray. Mathematical biology. Springer-Verlag, New York, 1993.

[46] J.D. Murray. Mathematical biology II: Spatial models and biomedical applications. Springer-Verlag, New York, 2003.

[47] W. Nentwig (Ed.). Biological invasions. Ser. in Ecological studies. vol. 193. Springer, Berlin, 2007.

[48] A. Okubo, S.A. Levin. Diffusion and ecological problems: modern perspectives. Springer, New York, 2001. 
[49] B. Palmer, R.E.C. McFadyen. Ambrosia artemisiifolia L. - annual ragweed. in Biological control of weeds in Australia (M.H. Julien, R.E.C. McFadyen, J.M. Cullen, Eds.) CSIRO, Collingwood, Australia, 2012, 52-59.

[50] S.V. Petrovskii, B.L. Li. Exactly solvable models of biological invasion. CRC Press, Boca Raton, 2006.

[51] S.Y. Reznik, I.A. Spasskaya, M.Y. Dolgovskaya, M.G. Volkovitsh, V.F. Zaitzev. The ragweed leaf beetle Zygogramma suturalis F. (Coleoptera: Chrysomelidae) in Russia: current distribution, abundance and implication for biological control of common ragweed, Ambrosia artemisiifolia L. in Proceedings of the XII International Symposium on Biological Control of Weeds (M.H. Julien R. Sforza, M.C. Bon, H.C. Evans, P.E. Hatcher, H.L. Hinz, B.G. Rector, Eds.), CABI, Wallingford, UK, 2008, 614-619.

[52] P.M. Room. Ecology of a simple plant-herbivore system. Biological control of Salvinia. Trends Ecol. Evol., 5, (1990), No. 3, 74-79.

[53] P.M. Room, P.A. Thomas. Nitrogen and establishment of a beetle for biological control of the floating weed Salvinia in Papua New Guinea. J. Appl. Ecol., 22 (1985), 139-156.

[54] L. Roques, J. Garnier, F. Hamel, E.K. Klein. Allee effect promotes diversity in traveling waves of colonization. Proc. Nat. Acad. Sci. USA, 109 (2012), No. 23, 8828-8833.

[55] N. Sapoukhina, Yu. Tyutyunov, R. Arditi. The role of prey-taxis in biological control: a spatial theoretical model. Am. Nat., 162 (2003), No. 1, 61-76.

[56] W.E. Schiesser. The numerical method of lines: integration of partial differential equations. Academic Press, San Diego, 1991.

[57] E. Scholze, H. Pichler, H. Heran. Zur Entfernungsschätzung der Bienen nach dem Kraftaufwand. Naturwissenschaften, 51 (1964), 69-70.

[58] S.O. Sergievskii. Choosing of partner for copulation in populations of Zygogramma suturalis F. in: Theoretical Principles of Biological Control of the Common Ragweed (O.V. Kovalev, S.A. Belokobylsky, Eds.). Proceedings of the Zoological Institute. vol. 189. "Nauka" Publishing House, Leningrad Branch, Leningrad, 1989, 173-176. [in Russian]

[59] P.A. Stephens, W.J. Sutherland. Consequences of the Allee effect for behaviour, ecology and conservation. Trends Ecol. Evol., 14 (1999), 401-405.

[60] H.L. Sweetman. The Principles of Biological Control. W.C. Brown Co, Dubuque, Iowa, 1958.

[61] R. Tourniaire, A. Ferran, L. Giuge, C. Piotte, J. Gambier. A natural flightless mutation in the ladybird, Harmonia axyridis. Entomologia Experimentalis et Applicata, 96 (2000), 33-38.

[62] Yu.V. Tyutyunov, N.Yu. Sapoukhina, I.N. Senina, R. Arditi. Explicit model for searching behavior of predator. Zhurnal Obshchei Biologii, 63 (2002), No. 2, 137-148. [in Russian]

[63] Yu. Tyutyunov, I. Senina, R. Arditi. Clustering due to acceleration in the response to population gradient: a simple self-organization model. Am. Nat., 164 (2004), No. 6, 722-735.

[64] Yu. Tyutyunov, L. Titova, R. Arditi. A minimal model of pursuit-evasion in a predator-prey system. Math. Model. Nat. Phenom., 2 (2007), No. 4, 122-134.

[65] Yu. Tyutyunov, L. Titova, R. Arditi. Predator interference emerging from trophotaxis. Ecol. Complex., 5 (2008), No. $1,48-58$.

[66] Yu.V. Tyutyunov, A.D. Zagrebneva, F.A. Surkov, A.I. Azovsky. Microscale patchiness of the distribution of copepods (Harpacticoida) as a result of trophotaxis. Biophysics, 54 (2009), No. 3, 355-360.

[67] Yu. Tyutyunov, E. Zhadanovskaya, D. Bourguet, R. Arditi. Landscape refuges delay resistance of the European corn borer to Bt-maize: a demo-genetic dynamic model. Theor. Popul. Biol., 74 (2008), 138-146.

[68] Yu.V. Tyutyunov, E.A. Zhadanovskaya, R. Arditi, A.B. Medvinsky. A spatial model of the development of pest resistance to a transgenic insecticidal crop: European corn borer on Bt maize. Biophysics, 52 (2007), No. 1, 52-67.

[69] T.C.R. White. The inadequate environment: nitrogen and the abundance of animals. Springer, Berlin, 1993.

[70] T.C.R. White. Why does the world stay green?: nutrition and survival of plant-eaters. CSIRO Publishing, Collingwood, Australia, 2005.

[71] T. Yamanaka, K. Tanaka, A. Otuka, O.N. Bjørnstad. Detecting spatial interactions in the ragweed (Ambrosia artemissifolia L.) and the ragweed beetle (Ophraella communaLeSage) populations. Ecol. Res., 22 (2007), $185-196$.

[72] S.-R. Zhou, Y.-F. Liu, G. Wang. The stability of predator-prey systems subject to the Allee effects. Theor. Popul. Biol., 67 (2005), 23-31. 